\title{
On the edge of degradation: Autophagy regulation by RNA decay
}

\author{
Elizabeth Delorme-Axford ${ }^{1}$ and Daniel J. Klionsky ${ }^{1 \#}$
}

From the ${ }^{1}$ Life Sciences Institute, University of Michigan, Ann Arbor, MI 48109, USA

\# Correspondence to: Daniel J. Klionsky; University of Michigan; Life Sciences Institute; Rm. 6036; 210 Washtenaw Ave.; Ann Arbor, Michigan 48109-2216 USA;

Email: klionsky@umich.edu

Keywords: Dcp2, DDX6, Dhh1, mRNA decay, RNA degradation, vacuole, Xrn1/XRN1, yeast

Abbreviations: ATG, autophagy-related; CPA, cleavage and polyadenylation; Cvt, cytoplasmto-vacuole targeting; GABARAP, GABA type A receptor-associated protein; MAP1LC3/ LC3, microtubule associated protein 1 light chain 3; miRNA, microRNA; mRNA, messenger RNA; MTOR, mechanistic target of rapamycin kinase; NMD, nonsense-mediated decay; qRT-PCR, quantitative real-time PCR; SG, stress granule; siRNA, small interfering RNA; TOR, target of rapamycin; TORC1, TOR complex 1; Ubl, ubiquitin-like; UTR, untranslated region

This is the author manuscript accepted for publication and has undergone full peer review but has not been through the copyediting, typesetting, pagination and proofreading process, which may lead to differences between this version and the Version of Record. Please cite this article as doi: 10.1002/wrna.1522

This article is protected by copyright. All rights reserved. 
Running title: Autophagy regulation by RNA decay

\begin{abstract}
Cells must dynamically adapt to altered environmental conditions, particularly during times of stress, to ensure their ability to function effectively and survive. The macroautophagy/autophagy pathway is highly conserved across eukaryotic cells and promotes cell survival during stressful conditions. In general, basal autophagy occurs at a low level to sustain cellular homeostasis and metabolism. However, autophagy is robustly upregulated in response to nutrient deprivation, pathogen infection and increased accumulation of potentially toxic protein aggregates and superfluous organelles. Within the cell, RNA decay maintains quality control to remove aberrant transcripts and regulate appropriate levels of gene expression. Recent evidence has identified components of the cellular mRNA decay machinery as novel regulators of autophagy. Here, we review current findings that demonstrate how autophagy is modulated through RNA decay.
\end{abstract}

\title{
1. An Introduction to Autophagy
}

\subsection{Overview}

This article is protected by copyright. All rights reserved. 
When encountering altered environments and fluctuating nutrient conditions, cells must respond and adapt through mechanisms that support their survival. Autophagy is a precisely controlled catabolic process of cellular "self-eating" that is highly conserved across eukaryotes (from yeast to human). Starvation, pathogen infection and/or the accumulation of damaged or superfluous organelles can activate the autophagy pathway, which enables eukaryotic cells to function and survive amid stress conditions. Autophagy also removes harmful cellular material, while at the same time providing a source of macromolecules to sustain cellular metabolism during low-energy periods. The de novo formation of the sequestering phagophore, which matures into a double-membrane autophagosome is the characteristic morphological feature of autophagy. At present, 42 autophagy-related (ATG) genes have been identified in fungi; many of these genes have homologs or at least functional counterparts in higher eukaryotes.

Autophagy is primarily a degradative pathway requiring the vacuole (in yeast or plants) or lysosomes (in metazoans) for the breakdown of sequestered cargo. However, the biosynthetic cytoplasm-to-vacuole targeting (Cvt) pathway utilizes most of the autophagy machinery (Lynch-Day and Klionsky 2010), and secretory autophagy has been recently described as an alternate mechanism for unconventional protein secretion (Ponpuak et al. 2015). Briefly, canonical degradative autophagic flux includes 5 major steps (see Figure 1). Autophagy occurs constitutively, but enhanced autophagy is triggered by a stimulus such as nutrient deprivation or other types of stress including hypoxia, or by treatment with a pharmacological agent such as rapamycin or torin 1. During basal or induced autophagy, the phagophore 1) nucleates; 2) expands and 3) matures and closes to form the complete, doublemembrane autophagosome. The autophagosome 4) fuses with the degradative compartment 
and 5) the sequestered cargo undergoes degradation. The resulting macromolecules are released into the cytosol as anabolic building blocks or for catabolism to maintain cellular function.

There are 2 major forms of cellular autophagy—selective and nonselective-and the primary mode of autophagy is termed macroautophagy. Autophagy mediates the clearance of cargo that exceeds the physical and spatial capacity of the proteasome, the other major degradative mechanism in eukaryotic cells. Furthermore, emerging evidence indicates likely cross-talk between autophagy and the ubiquitin-proteasome system (which typically targets short-lived or misfolded proteins) (Kwon and Ciechanover 2017; Dikic 2017; Klionsky and Schulman 2014; Grumati and Dikic 2018).

Although autophagy was initially characterized as being non-selective, the molecular details of such a process are not clear. For example, how does the phagophore, the initial sequestering compartment, nucleate in the absence of specific cargo? Are parts of the cell randomly sequestered, or is there some type of segregation such as a phase separation step that initially segregates portions of the cytoplasm? Without specific cargo, how does the phagophore achieve a particular curvature, which will determine the size of the autophagosome? Even for proteins within organelles such as mitochondria, degradation apparently occurs in a hierarchical manner (Dengjel, Kristensen, and Andersen 2008; Kristensen et al. 2008). In selective autophagy, cargo is targeted through receptor-mediated interactions and physical proximity to the elongating phagophore (Sawa-Makarska et al. 2014). Examples of selective autophagy include but are not limited to: the Cvt pathway, which has only been identified in fungi (Harding et al. 1995; Scott et al. 2001; Yorimitsu and Klionsky 2005; 
Farre, Vidal, and Subramani 2007), mitophagy (Kanki et al. 2009; Okamoto, Kondo-Okamoto, and Ohsumi 2009), pexophagy (Hutchins, Veenhuis, and Klionsky 1999), reticulophagy (Bernales, McDonald, and Walter 2006; Mochida et al. 2015), ribophagy (Kraft et al. 2008) and granulophagy (Buchan et al. 2013).

Recently there has been a call to classify autophagy based on its participation in metabolism versus quality control, rather than as selective versus non-selective. Metabolic autophagy is coupled with induction by nutrient deprivation and with non-selective autophagy. Both quality control and metabolic autophagy function in a complementary capacity, such as during mitophagy. Prolonged growth in media containing a non-fermentable carbon source increases mitochondrial proliferation in yeast. When these cells are shifted to nitrogen-free (starvation) medium containing glucose, cells undergo mitophagy to eliminate excess mitochondria. Alternatively, culturing yeast cells to post-log phase for several days in media containing a non-fermentable carbon source can also induce mitophagy; this is known as "stationary phase mitophagy" (Delorme-Axford et al. 2015; Guimaraes et al. 2015). For additional review on the mechanisms of selective autophagy, see refs. (Gatica, Lahiri, and Klionsky 2018; Anding and Baehrecke 2017).

Given the significant role that autophagy plays in cell health and the need for precise mechanisms to strictly moderate its activity in the cell, abnormal autophagy activity can be highly detrimental and is associated with human diseases such as cancer, neurodegeneration and lysosomal storage disorders. Pharmaceutical targeting of autophagy is currently being pursued in the clinic, particularly in the fields of cancer therapeutics and neurodegenerative diseases (reviewed in (Thorburn 2018)). Stimulation of autophagy has also been attributed to 
the metabolic and physiological benefits associated with exercise (He et al. 2012). Despite decades of research, the vast complex regulatory network modulating autophagy within the cell is still under active investigation. Thus, it is of critical importance to more fully understand the dynamic network controlling the autophagy response, not only in yeast, but also across eukaryotic cells. Cells regulate the magnitude of the autophagy response by controlling the size (Xie, Nair, and Klionsky 2008; Cawthon et al. 2018) and/or number (Jin et al. 2014; Cawthon et al. 2018) of autophagosomes formed. The cell has also evolved mechanisms to control autophagy at various stages of Atg protein synthesis and function, including at the transcriptional, post-transcriptional, translational and post-translational levels. Below we will concisely describe the components and processes involved in each stage of the autophagy pathway before reviewing relevant work on its post-transcriptional regulation by cellular mRNA decay, with an emphasis on yeast as a model system for this work.

\subsection{Components and main stages of autophagy in the yeast Saccharomyces cerevisiae}

Autophagy is commonly activated through nutrient starvation or by treatment with rapamycin in yeast (Delorme-Axford et al. 2015). In mammalian cells, amino acid starvation, glucose starvation or pharmacological agents are routinely used to induce autophagy. Rapamycin inhibits TOR (target of rapamycin) in yeast (or MTOR [mechanistic target of rapamycin kinase] in mammalian cells), a serine/threonine kinase that is a critical negative regulator of autophagy (Noda and Ohsumi 1998); although MTOR-independent mechanisms have also been described (Mordier et al. 2000; Sarkar et al. 2007). In yeast, the perivacuolar phagophore assembly site (PAS) is a single punctum that will eventually form the complete 
autophagosome (Kirisako et al. 1999). In mammalian cells, it is generally accepted that the phagophore (or the nascent forming autophagosome membrane) is generated at sites termed the omegasome derived from the endoplasmic reticulum; however, input of membrane from other organelles and structures appear to be involved as well (Axe et al. 2008; Yu, Chen, and Tooze 2018).

Beginning with the Atg1 complex (which includes Atg1, Atg13 and the Atg17-Atg31Atg29 ternary subcomplex), the "core" Atg components assemble at the PAS in a hierarchal order (Table 1 and ref. (Parzych and Klionsky 2014; Mao et al. 2013)). Subsequent to recruitment and assembly of the Atg1 complex, Atg9 (along with Atg2 and Atg18) localize to the PAS (Feng et al. 2014). Atg9 is an integral membrane protein involved in supplying membrane for the expanding phagophore, and cycles between the PAS and peripheral sites (Reggiori et al. 2005; Feng et al. 2016; Gomez-Sanchez et al. 2018). At these peripheral sites, Atg23 and Atg27 function in the formation of Atg9-containing vesicles (Backues et al. 2015). Levels of Atg9 directly control the frequency of autophagosome formation (Jin et al. 2014). Expansion of the phagophore to form the mature double-membrane autophagosome requires 2 conserved ubiquitin-like (Ubl) conjugation systems, involving Atg12 and Atg8. The Atg12 Ubl system (consisting of Atg5, Atg7, Atg10, Atg12 and Atg16) supports the formation of the Atg12-Atg5Atg16 heterotrimeric complex, which functions as an E3-like enzyme for the Atg8 conjugation system (Mizushima, Noda, and Ohsumi 1999; Kuma et al. 2002; Hanada et al. 2007; SakohNakatogawa et al. 2013). At the phagophore, the covalent conjugation of Atg12 to Atg5 requires Atg7, an E1-like enzyme that activates Atg12, and the E2-like enzyme Atg10 (Mizushima et al. 1998; Shintani et al. 1999; Tanida et al. 1999). The Atg8 Ubl system is required for the 
expansion and closure of the phagophore to form the complete autophagosome, and requires Atg3, Atg4, Atg7 and Atg8 (Feng et al. 2014). Nascent Atg8 undergoes proteolytic processing at its $C$ terminus by Atg4 (Kirisako et al. 2000). The processed Atg8 is subsequently activated by Atg7, and conjugated to phosphatidylethanolamine (PE) by Atg3, a second E2-like enzyme, to generate Atg8-PE (Kirisako et al. 2000; Kim, Huang, and Klionsky 2001; Ichimura et al. 2000). Levels of Atg8 and Atg7 directly correlate with the size of autophagosomes formed (Xie, Nair, and Klionsky 2008; Cawthon et al. 2018). MAP1LC3/LC3 (microtubule associated protein 1 light chain 3) and GABARAP (GABA type A receptor-associated protein) isoforms form 2 subfamilies of mammalian homologs of yeast Atg8 (Kabeya et al. 2000); however, no studies have examined the role of these proteins in controlling the size of the autophagosome. Similarly, there is no information available on the role of mammalian ATG9 homologs in regulating the frequency of autophagosome formation.

Next, the autophagosome undergoes docking and fusion with the vacuole or lysosome; as with other fusion events, Rab and SNARE (soluble N-ethylmaleimide-sensitive factor attachment protein receptor) proteins are involved. In yeast, Atg17 (as part of the ternary Atg17Atg31-Atg29 subcomplex) interacts with Atg11 to recruit the SNARE protein Vam7 to facilitate fusion of the autophagosome with the vacuole (Liu et al. 2016). Following fusion, the autophagosome inner vesicle is released as an autophagic body in the vacuole lumen; these structures are not observed in mammalian cells due to the relatively small size of the mammalian lysosome. Finally, the remaining cargos undergo degradation by vacuolar or Iysosomal hydrolases. At least for yeast, the lipase Atg15 is required for the degradation of Cvt vesicle and autophagic body membranes within the vacuole (Teter et al. 2001; Epple et al.

This article is protected by copyright. All rights reserved. 
2001), and a mechanism for the efflux of amino acids from the vacuole (involving the vacuolar integral membrane protein Atg22 among other transporters) has been identified (Yang et al. 2006). Nonselective degradation and efflux of RNA in yeast seems to be inefficient, and nucleobases appear to be excreted by the cell (Huang et al. 2015). Currently the mechanism(s) by which other nucleic acids, lipid, and/or carbohydrates are recycled have yet to be identified. For a more comprehensive review on induction and flux through the autophagy pathway, see refs. (Wen and Klionsky 2016; Mercer, Gubas, and Tooze 2018; Reggiori and Ungermann 2017).

\section{The relationship between autophagy and RNA regulatory mechanisms}

\subsection{Clearance of RNA by autophagy}

Proper maintenance of RNA metabolism is critical to maintain cellular health. The cell must strike a critical balance between the synthesis, localization, folding, modification and decay of RNA. Emerging evidence for the interplay between autophagy and RNA degradation pathways has become increasingly clear in recent years, with work demonstrating roles for autophagy in RNA degradation and the reciprocal control of autophagy by the cellular RNA decay machinery (described further in section 3 and reviewed in (Frankel, Lubas, and Lund 2017)). Below, we highlight current findings supporting this mutual regulation.

\subsection{RNA degradation by non-selective starvation-induced autophagy}

Recently, a role for autophagy has been described in the degradation of bulk RNA

This article is protected by copyright. All rights reserved. 
through starvation-dependent non-selective autophagy in yeast (Huang et al. 2015). The T2 family ribonuclease Rny1 is involved in the initial RNA cleavage events (in the vacuole), producing 3' nucleotides (Huang et al. 2015). These RNA degradation products are further processed by the phosphatase Pho8, resulting in the accumulation of nucleosides in the vacuole (Huang et al. 2015). These RNA metabolites are transported out of the vacuole and further catabolized by the nucleosidases Pnp1 and Urh1 in the cytosol (Huang et al. 2015); although the mechanism and the transporter(s) involved have yet to be identified. The resulting majority of nucleobases are then inefficiently secreted from the cell (Huang et al. 2015). The mechanism for bulk RNA degradation is also conserved in the fission yeast Schizosaccharomyces pombe (Huang et al. 2015). This autophagy-dependent pathway for RNA degradation is a bit of a paradox - the cell apparently goes to great lengths to ensure that these macromolecules are available, and yet instead of reusing the nucleobases, the cell excretes them. Future studies will determine the specific details of RNA processing in the yeast vacuole, and the homeostatic and metabolic benefits to the cell for this seemingly costly form of RNA catabolism.

\subsection{Ribophagy: a selective autophagy mechanism targeting ribosomes}

Ribophagy, a selective starvation-dependent mechanism for the removal of ribosomes, has been described in yeast (Kraft et al. 2008). The selective degradation of ribosomes requires the ubiquitin protease Ubp3 and its cofactor Bre5, and cells deficient in Ubp3-Bre5 demonstrate increased substrate ubiquitination, supporting a role for Ubp3-Bre5 deubiquitination during ribophagy (Kraft et al. 2008). Genetic deletion of UBP3 or BRE5 does not affect non-selective 
autophagy, but does indicate a role for both non-selective autophagy in the uptake of mature ribosomes and selective uptake of $40 S$ and 605 ribosomal subunits (Kraft et al. 2008). Another report indicates that the chaperone-like protein Cdc48 and Doa1/Ufd3 (a ubiquitin binding cofactor of Cdc48) are also required for ribophagy in yeast (Ossareh-Nazari et al. 2010). A later study identified the E3 ubiquitin ligase Rkr1/Ltn1 as a negative regulator of starvationdependent Ubp3-mediated ribophagy through ubiquitination of the $60 \mathrm{~S}$ ribosomal protein Rpl25 (Ossareh-Nazari et al. 2014). However, a comparable mechanism involving metazoan homologs has yet to be identified.

A recent study from the Sabatini lab identified NUFIP1 (NUFIP1, FMR1 interacting protein 1) in mammalian cells as the selective ribosome receptor during starvation-induced ribophagy through quantitative proteomic analysis of purified lysosomes (Wyant et al. 2018); NUFIP1 is also required for efficient starvation-induced ribophagy (Wyant et al. 2018). NUFIP1 forms a heterodimer with ZNHIT3 (zinc finger HIT-type containing 3) (Quinternet et al. 2016; Rothe et al. 2014). NUFIP1-ZNHIT3 accumulate on autophagosomes when MTOR complex 1 is inhibited and bind LC3B through a confirmed LC3-interacting region (Wyant et al. 2018). It is estimated that $\sim 50 \%$ and $\sim 80 \%$ of total cellular protein and RNA, respectively, are associated with ribosomes (Wyant et al. 2018). Selectively targeting ribosomes during conditions in which nutrients are scare would thus provide a rich pool of potential macromolecular building blocks during prolonged starvation periods.

\subsection{Granulophagy: Stress granule clearance through autophagy}

This article is protected by copyright. All rights reserved. 
Stress granules (SGs) form during times of stress when translation initiation is restricted, and are transient hubs of mRNA, messenger RNA ribonuclear complex and RNA-binding protein storage (see (Sheinberger and Shav-Tal 2017; Protter and Parker 2016) for additional reviews). Stress granules (SGs) can be targeted to the vacuole through autophagy in a selective process termed "granulophagy" in both yeast and mammalian cells (Buchan et al. 2013). In a study by Buchan et al., stress granules are targeted for autophagy degradation in a process that requires Cdc48 in yeast and its ortholog VCP (valosin containing protein) in mammalian cells (Buchan et al. 2013). In yeast, these SGs are degraded within the vacuole in a process that is dependent on the vacuolar lipase Atg15 (Buchan et al. 2013). Simultaneous deletion of ATG15 and genes encoding canonical 5' to 3' mRNA decay components-the decapping factor Dcp2 and the exonuclease $\mathrm{Xrn} 1$-increase the frequency of SG formation in yeast, also resulting in greater accumulation of the SG marker Pab1 in the vacuole (Buchan et al. 2013). Granulophagy occurs in the absence of autophagy induction (i.e., under nutrient-replete conditions following stress induction and SG formation) (Buchan et al. 2013), indicating a role for a quality control autophagy mechanism. In mammalian cells, the clearance of SGs is diminished when autophagy is inhibited or when VCP is depleted (Buchan et al. 2013). It is intriguing that both stress-induced granulophagy (Buchan et al. 2013) and starvation-induced ribophagy require Cdc48 (Ossareh-Nazari et al. 2010) for autophagy-mediated degradation. Thus far, no selective autophagy receptor has been identified for granulophagy in either yeast or mammalian cells.

\subsection{Retrotransposons are degraded by autophagy}

This article is protected by copyright. All rights reserved. 
Retrotransposons are transposable elements that integrate into the genome through a cytoplasmic RNA intermediate (reviewed in (Elbarbary, Lucas, and Maquat 2016)). In S. cerevisiae, the Ty1 retrotransposon is the most abundant retrotransposon in yeast and inserts its cDNA into the genome upon entering the nucleus (Rowley 2017; Suzuki et al. 2011). During nutrient-rich conditions, Ty1 virus-like particles are transported from the cytoplasm to the vacuole through the biosynthetic Cvt pathway (Suzuki et al. 2011). During starvation conditions, Ty1 virus-like particles are trafficked to the vacuole through a selective autophagy mechanism involving the Cvt pathway cargo receptor Atg19 (Suzuki et al. 2011; Scott et al. 2001). Suzuki and colleagues propose that this mechanism may function to moderate cellular genomic change during nutrient-limiting conditions (Suzuki et al. 2011).

Similarly, autophagy is implicated in the clearance of retrotransposon RNA from both long and short interspersed elements in mammalian cells (Guo et al. 2014). Guo et al. reported that retrotransposon RNA colocalizes and copurifies with autophagosomes (delineated with the autophagosome marker LC3) (Guo et al. 2014). Autophagy-dependent targeting of longinterspersed nucleotide elements RNA is dependent on the autophagy receptors CALCOCO2/NDP52 (calcium binding and coiled-coil domain 2) and SQSTM1/p62 (Guo et al. 2014). Autophagy inhibition occurs in mice that are partially deficient in BECN1/Vps30 (Guo et al. 2014). These Becn $1^{+/-}$mice show increased accumulation of cytoplasmic retrotransposon RNA and genomic insertions, further supporting the idea that autophagy may function in this mechanism to promote genome stability and mitigate evolutionary change (Guo et al. 2014). Although various RNA species have been identified as cargo for degradation through either 
non-selective or selective autophagy pathways, future studies will determine if additional RNA elements that have yet to be identified may be targeted through these pathways.

\subsection{Mammalian microRNA (miRNA) processing components are targeted by autophagy}

In mammals, non-coding RNAs including microRNAs (miRNAs) are key post-

transcriptional regulators of gene expression (see (Bartel 2018)) . Recent work has identified a selective autophagy mechanism to regulate miRNA activity (Gibbings et al. 2012). The RNA interference pathway components DICER1 (an miRNA-processing enzyme) and AGO2 (the main miRNA effector) are cleared by selective autophagy, thereby modulating miRNA activity (Gibbings et al. 2012). Ubiquitin-dependent CALCOCO2 was identified as the selective autophagy receptor for DICER1 (Gibbings et al. 2012).

\section{Autophagy regulation by RNA decay}

RNA decay mechanisms function to ensure that gene expression is maintained at appropriate levels to meet cellular needs while at the same time maintain quality control to regulate and remove aberrant transcripts from the cell. The 2 major pathways of RNA decay include degradation in either the $5^{\prime}$ to $3^{\prime}$ or the $3^{\prime}$ to $5^{\prime}$ direction (Figure 2). In yeast, canonical mRNA degradation typically occurs following shortening of the poly[A] tail (also referred to as deadenylation) by the Pan2-Pan3 and Pop2-Ccr4-Not complexes, followed by decay in either the $5^{\prime}$ to $3^{\prime}$ or the or the $3^{\prime}$ to $5^{\prime}$ direction (Parker 2012; Labno, Tomecki, and Dziembowski 2016). In 5' to 3' RNA decay, the Dcp2 decapping complex removes the 5' methylguanosine cap, exposing 5'-monophosphate; the cytoplasmic exonuclease Xrn1 hydrolyzes the transcript.

This article is protected by copyright. All rights reserved. 
The multi-subunit RNA exosome complex mediates degradation in the 3' to 5' direction. Other cytoplasmic RNA decay mechanisms also exist, including nonsense-mediated decay (NMD; further described below in section 3.2), no-go decay (Doma and Parker 2006) and non-stop decay (van Hoof et al. 2002; Frischmeyer et al. 2002). To note, much of the work that has been done to elucidate these pathways has come primarily from S. cerevisiae (Huch and Nissan 2014). For a more comprehensive review on the detailed mechanisms governing RNA decay pathways, see (Parker 2012; Heck and Wilusz 2018; Labno, Tomecki, and Dziembowski 2016).

\subsection{5' to 3' mRNA decay factors that regulate autophagy}

\subsubsection{The decapping factor Dcp2 and RCK family RNA helicases Dhh1/Vad1/DDX6}

The yeast kinase Rim15 positively regulates autophagy through the integration of signals from 2 major nutrient sensing pathways_TOR and protein kinase A (Yorimitsu et al. 2007; Delorme-Axford and Klionsky 2018; Reggiori and Klionsky 2013). Rim15 phosphorylates at least 2 mediators of ATG gene repression in yeast-Ume6 (Bartholomew et al. 2012) and Rph1 (Bernard, Jin, Gonzalez-Rodriguez, et al. 2015). Additionally, Rim15 phosphorylates the paralogous proteins Igo1 and Igo2 when TOR complex 1 (TORC1) is inhibited by the pharmacological agent rapamycin (Talarek et al. 2010). Igo1/2 are required for cells to enter the reversible quiescent $\left(G_{0}\right)$ program of the cell cycle characterized by a state of low metabolic activity (Talarek et al. 2010). In this mechanism, the phosphorylation of Igo1/2 by Rim15 leads to their association with the mRNA decapping activator Dhh1 (Talarek et al. 2010). The association of Igo1/2 with Dhh1 protects transcripts from RNA decay, thereby promoting their translation when TORC1 is inhibited (Talarek et al. 2010). Through this mechanism, the cell

This article is protected by copyright. All rights reserved. 
protects mRNAs from the RNA decay pathway during nutrient-limiting conditions that promote autophagy (Talarek et al. 2010). Thus, Igo1/2 phosphorylation promotes interaction with the decapping coactivator Dhh1 to facilitate transcript protection from 5' to 3' degradation in a TORC1-dependent manner (Talarek et al. 2010); however, whether Igo1/2-Dhh1 are involved in the protection of ATG transcripts during starvation-induced autophagy remains to be investigated.

Further linking the complex relationship between autophagy regulation and the RNA decay machinery, nitrogen starvation in yeast induces the rapid depletion in a Ubp3-dependent manner of several factors important for RNA decay and translation, including: Dcp2 (a decapping factor), Pop2 (a deadenylation factor), Tif4631/eiF4G1 (a translation initiation factor) and Sup35/eRF3 (a translation termination factor) (Kelly and Bedwell 2015). The depletion of the decapping enhancer Pat1 and Pab1 (a poly[A] binding protein that stimulates deadenylation) occur in an autophagy-dependent manner (Kelly and Bedwell 2015). Dcp2 and Pop2 are degraded in a manner that is dependent on the proteasome and in response to rapamycin treatment, further supporting the involvement of TORC1 (Kelly and Bedwell 2015).

More recently, Hu et al. identified Dcp2-the catalytic subunit of the 5' to 3' mRNA decay decapping complex-and RCK family RNA-binding proteins as post-transcriptional regulators of autophagy, ATG mRNAs and autophagy-dependent innate immunity in yeast and mammalian cells (Hu et al. 2015). RCK family members are RNA helicases (lacking classical helicase activity (Presnyak and Coller 2013)) that include conserved homologs-Dhh1 in S. cerevisiae, Vad1 in Cryptococcus neoformans and DDX6/p54 in mammals. Dhh1 and other RCK proteins have been implicated as translational mediators (Presnyak and Coller 2013), although the 
precise mechanisms by which this occurs remain to be defined. However, it is thought that RCK family members interact with mRNAs through recruiting and binding the 5' untranslated region (UTR) of target transcripts (reviewed in (Presnyak and Coller 2013; Wen and Klionsky 2016)). Additionally, Dhh1 physically interacts with the decapping complex catalytic factor Dcp2 (Decker, Teixeira, and Parker 2007).

Strains lacking $D H H 1$ or harboring a temperature-sensitive (ts) mutation of $D C P 2$ demonstrate significant upregulation of ATG transcripts (Hu et al. 2015). In dhh1 cells, increased levels of $A T G 3,7,8,19,20,22$ and 24 mRNA are observed under nutrient-replete conditions (Hu et al. 2015). In rich conditions, transcript levels for ATG1 through ATG9, ATG11, ATG13 through ATG24, ATG29, 31, 32 and 34 are greatly enhanced (Hu et al. 2015). When starved for nitrogen, the $d h h 1 \Delta$ and $d c p 2-7 \Delta$ ts strains exhibit higher levels of autophagy activity when assessed by multiple assays (Hu et al. 2015). The regulation of ATG genes through RCK family members is highly conserved from yeast ( $S$. cerevisiae and pathogenic $C$. neoformans) to mammalian cells (Hu et al. 2015). Decapping of ATG mRNAs, especially ATG8, is mediated by the Dhh1 homolog Vad1 in C. neoformans (Hu et al. 2015). Under nutrient-replete conditions, Dcp2 is phosphorylated by TORC1 in C. neoformans, driving its regulation of (at least) ATG8 mRNA by recruitment to the decapping machinery (Hu et al. 2015). In this mechanism, TORC1 negatively regulates the translation of $A T G$ transcripts under nutrient-replete conditions, but promotes the translation of the bulk of cellular transcripts (Delorme-Axford and Klionsky 2018). A homologous mechanism was identified in mammalian cells wherein MTOR phosphorylates DDX6 (Hu et al. 2015). Subsequently, the MAP1LC3 transcript undergoes decapping (and is presumably targeted for RNA degradation) under fed conditions, thereby inhibiting autophagy

This article is protected by copyright. All rights reserved. 
(Hu et al. 2015). The downregulation of $A T G$ transcripts under nutrient-rich conditions maintains autophagy at a basal level. During starvation, TORC1 is inactivated, facilitating translation (rather than degradation) of these transcripts, further supporting autophagy progression.

\subsubsection{The exonuclease Xrn1}

Downstream of Dhh1 and Dcp2 in the canonical 5' to 3' mRNA decay pathway, the exonuclease Xrn1 is highly conserved and hydrolyzes decapped transcripts. Xrn1/XRN1 is a post-transcriptional negative regulator of autophagy in yeast and mammalian cells (DelormeAxford et al. 2018). In the absence of $X R N 1$, cells induce a more rapid and robust autophagy response when starved for nitrogen (Delorme-Axford et al. 2018). Cells lacking XRN1 display an increased frequency (but not size) of autophagosome formation when analyzed by transmission electron microscopy (Delorme-Axford et al. 2018). Under nutrient-replete conditions, select ATG transcripts are upregulated including $A T G 1,4,5,7,8,12,14,16,29$ and 31 in $x r n 1 \Delta$ cells when assessed by quantitative real-time PCR (qRT-PCR) (Delorme-Axford et al. 2018). The ribonuclease activity of $\mathrm{Xrn} 1$ is required for the regulation of (at least) ATG8, 12 and 29 during nutrient-rich conditions (Delorme-Axford et al. 2018). Xrn1 mRNA and protein levels are markedly diminished when cells are starved for nitrogen in wild-type cells, further supporting its role as a negative autophagy regulator (Delorme-Axford et al. 2018).

Due to the high conservation of Xrn1/XRN1 in mammalian cells, the impact on autophagy was examined in mammalian cells by reducing XRN1 expression with small interfering RNA (siRNA) (Delorme-Axford et al. 2018). Autophagy flux was upregulated in a starvation-independent manner when examined by multiple assays in mammalian cells

This article is protected by copyright. All rights reserved. 
(Delorme-Axford et al. 2018). In BECN1 CRISPR knockout (KO) cells, the enhanced autophagy phenotype was lost, supporting the involvement of the canonical autophagy pathway (DelormeAxford et al. 2018). BECN1 is a subunit of the phosphatidylinositol 3-kinase complex I required for autophagy induction, and is the mammalian ortholog of yeast Vps30/Atg6 (Liang et al. 1999). Depletion of XRN1 in mammalian cells is associated with increased poliovirus replication through an autophagy-dependent mechanism (Delorme-Axford et al. 2018), supporting the conservation of Xrn1/XRN1 as a negative autophagy regulator. Poliovirus (and other picornaviruses) require host membranes for viral genome replication; these membranes are thought to be derived from autophagosomes (Jackson et al. 2005; Wong et al. 2008).

Tang et al. identified TORC1 regulation in RNA processing and modulation of Atg transcripts through the cleavage and polyadenylation (CPA) complex in Drosophila (Tang et al. 2018). Nutrient status (fed versus starved) and the CPA complex regulate 3' UTR length and alternative splicing of Atg1 and Atg8a transcripts (Tang et al. 2018). Starvation and the CPA complex result in longer 3' UTR lengths, which is associated with improved transcript stability and enhanced protein expression (Tang et al. 2018). The data provided by Tang et al. (Tang et al. 2018) further support the role of additional layers of gene regulation involved in controlling the magnitude of the autophagy response and provide a paradigm for further investigation into the role of UTRs and alternative splicing of ATG transcripts in autophagy modulation.

Although recent work has shed light on the regulation of autophagy through RNA decay mechanisms, intriguing questions remain, especially regarding the differential targeting of transcripts by Dhh1, Dcp2 and Xrn1. Are there other regulatory factors (in addition to Dhh1) involved in activating Dcp2 that may mediate selective transcript targeting (either stimulatory or 
inhibitory) to differentially regulate autophagy post-transcriptionally? What are the sequencespecific elements mediating the regulation of these ATG transcripts? Components of the 5' to 3' decay pathway (Dcp2, Dhh1 and Xrn1) have been implicated as post-transcriptional regulators of autophagy under nutrient-rich conditions. However, whether there are additional RNA decay mechanisms controlling autophagy such as the 3' to 5' exosome-mediated pathway or other minor degradation mechanisms (either during nutrient-rich, starvation or selective conditions) has yet to be fully explored.

\subsection{Nonsense-mediated decay (NMD) pathway}

NMD was first described as a surveillance mechanism for targeting and degrading mRNA transcripts with premature translation termination codons (Losson and Lacroute 1979). More recently, it has become generally accepted that NMD also regulates normal transcripts; although the mechanisms by which this selection occurs are still under investigation (Parker 2012; He and Jacobson 2015; Lykke-Andersen and Jensen 2015). Targeting by NMD requires 3 conserved factors, Nam7/Upf1/UPF1, Nmd2/Upf2/UPF2 and Upf3/UPF3 (reviewed in (He and Jacobson 2015)). In a mammalian cell line, knockdown of UPF1 and UPF2 by short hairpin RNAs (shRNAs) increases autophagy during basal, amino acid starvation and rapamycintreated conditions as measured by multiple assays (Wengrod et al. 2013). In contrast, autophagy is inhibited when NMD is hyperactivated (Wengrod et al. 2013). Wengrod et al. identified the NMD target ATF4 (a mammalian homolog of S. cerevisiae Gcn4, a major transcriptional regulator) as the mediator of autophagy induction during NMD inhibition (Wengrod et al. 2013). Cell viability is diminished when both the autophagy and NMD pathways

This article is protected by copyright. All rights reserved. 
are suppressed (Wengrod et al. 2013), supporting the importance of cellular programs for maintaining RNA quality control. To date, no such homologous mechanism(s) involving NMD factors has been identified as having an impact on autophagy in yeast.

\section{Conclusions}

Here we present an overview of what is currently known regarding autophagy regulation by cellular mRNA decay pathways (see Figure 3 for a summary). The eukaryotic cell has evolved multiple layers of regulation to maintain autophagy at a level that is appropriate to accommodate cellular needs. The myriad of mechanisms that are exerted by the cell to control the induction and magnitude of the autophagy response, along with the high level of pathway conservation throughout eukaryotes, highlight how important this process is for maintaining cellular integrity and survival during stress. Although here we focus mainly on posttranscriptional regulation by RNA decay, cells also utilize multiple layers of regulation through transcriptional (Bartholomew et al. 2012; Bernard, Jin, Gonzalez-Rodriguez, et al. 2015; Bernard, Jin, Xu, et al. 2015; Jin et al. 2014), translational (Wek, Zhu, and Wek 1995; RojasRios et al. 2015; Lubas et al. 2018) and post-translational (Feng et al. 2016; Mao et al. 2013; Russell et al. 2013) mechanisms (and reviewed in refs. (Delorme-Axford and Klionsky 2018; Feng, Yao, and Klionsky 2015; Popelka and Klionsky 2015)). Despite the immense progress that has been achieved towards understanding how cells control autophagy, many questions remain. The identification and characterization of novel factors involved in the selective degradation of distinct ATG transcripts by RNA decay and investigation into the post-

This article is protected by copyright. All rights reserved. 
transcriptional regulation of autophagy by non-canonical RNA decay pathways are areas for

future inquiry.

\section{Acknowledgements}

The authors apologize to those whose work was not included here due to space limitations.

D.J.K. is supported by funding from the National Institutes of Health (GM053396).

Conflict of Interest: The authors declare that no competing interests exist.

Anding, A. L., and E. H. Baehrecke. 2017. 'Cleaning House: Selective Autophagy of Organelles', Dev Cell, 41: 10-22.

Axe, E. L., S. A. Walker, M. Manifava, P. Chandra, H. L. Roderick, A. Habermann, G. Griffiths, and N. T. Ktistakis. 2008. 'Autophagosome formation from membrane compartments enriched in phosphatidylinositol 3-phosphate and dynamically connected to the endoplasmic reticulum', J Cell Biol, 182: 685-701.

Backues, S. K., D. P. Orban, A. Bernard, K. Singh, Y. Cao, and D. J. Klionsky. 2015. 'Atg23 and Atg27 act at the early stages of Atg9 trafficking in S. cerevisiae', Traffic, 16: 172-90.

Bartel, D. P. 2018. 'Metazoan MicroRNAs', Cell, 173: 20-51.

Bartholomew, C. R., T. Suzuki, Z. Du, S. K. Backues, M. Jin, M. A. Lynch-Day, M. Umekawa, A. Kamath, M. Zhao, Z. Xie, K. Inoki, and D. J. Klionsky. 2012. 'Ume6 transcription factor is part of a signaling cascade that regulates autophagy', Proc Natl Acad Sci U S A, 109: 11206-10.

Bernales, S., K. L. McDonald, and P. Walter. 2006. 'Autophagy counterbalances endoplasmic reticulum expansion during the unfolded protein response', PLoS Biol, 4: e423.

Bernard, A., M. Jin, P. Gonzalez-Rodriguez, J. Fullgrabe, E. Delorme-Axford, S. K. Backues, B. Joseph, and D. J. Klionsky. 2015. 'Rph1/KDM4 mediates nutrient-limitation signaling that leads to the transcriptional induction of autophagy', Curr Biol, 25: 546-55.

Bernard, A., M. Jin, Z. Xu, and D. J. Klionsky. 2015. 'A large-scale analysis of autophagyrelated gene expression identifies new regulators of autophagy', Autophagy, 11: 2114-22.

Buchan, J. R., R. M. Kolaitis, J. P. Taylor, and R. Parker. 2013. 'Eukaryotic stress granules are cleared by autophagy and Cdc48/VCP function', Cell, 153: 1461-74.

This article is protected by copyright. All rights reserved. 
Cawthon, H., R. Chakraborty, J. R. Roberts, and S. K. Backues. 2018. 'Control of autophagosome size and number by Atg7', Biochem Biophys Res Commun.

Decker, C. J., D. Teixeira, and R. Parker. 2007. 'Edc3p and a glutamine/asparagine-rich domain of Lsm $4 \mathrm{p}$ function in processing body assembly in Saccharomyces cerevisiae', J Cell Biol, 179: 437-49.

Delorme-Axford, E., E. Abernathy, N. J. Lennemann, A. Bernard, A. Ariosa, C. B. Coyne, K. Kirkegaard, and D. J. Klionsky. 2018. 'The exoribonuclease Xrn1 is a posttranscriptional negative regulator of autophagy', Autophagy, 14: 898-912.

Delorme-Axford, E., R. S. Guimaraes, F. Reggiori, and D. J. Klionsky. 2015. 'The yeast Saccharomyces cerevisiae: an overview of methods to study autophagy progression', Methods, 75: 3-12.

Delorme-Axford, E., and D. J. Klionsky. 2018. 'Transcriptional and post-transcriptional regulation of autophagy in the yeast Saccharomyces cerevisiae', J Biol Chem, 293: 5396-403.

Dengjel, J., A. R. Kristensen, and J. S. Andersen. 2008. 'Ordered bulk degradation via autophagy', Autophagy, 4: 1057-9.

Dikic, I. 2017. 'Proteasomal and Autophagic Degradation Systems', Annu Rev Biochem, 86: 193-224.

Doma, M. K., and R. Parker. 2006. 'Endonucleolytic cleavage of eukaryotic mRNAs with stalls in translation elongation', Nature, 440: 561-4.

Elbarbary, R. A., B. A. Lucas, and L. E. Maquat. 2016. 'Retrotransposons as regulators of gene expression', Science, 351: aac7247.

Epple, U. D., I. Suriapranata, E. L. Eskelinen, and M. Thumm. 2001. 'Aut5/Cvt17p, a putative lipase essential for disintegration of autophagic bodies inside the vacuole', $J$ Bacteriol, 183: 5942-55.

Farre, J. C., J. Vidal, and S. Subramani. 2007. 'A cytoplasm to vacuole targeting pathway in P. pastoris', Autophagy, 3: 230-4.

Feng, Y., S. K. Backues, M. Baba, J. M. Heo, J. W. Harper, and D. J. Klionsky. 2016. 'Phosphorylation of Atg9 regulates movement to the phagophore assembly site and the rate of autophagosome formation', Autophagy, 12: 648-58.

Feng, Y., D. He, Z. Yao, and D. J. Klionsky. 2014. 'The machinery of macroautophagy', Cell Res, 24: 24-41.

Feng, Y., Z. Yao, and D. J. Klionsky. 2015. 'How to control self-digestion: transcriptional, post-transcriptional, and post-translational regulation of autophagy', Trends Cell Biol, 25: 354-63.

Frankel, L. B., M. Lubas, and A. H. Lund. 2017. 'Emerging connections between RNA and autophagy', Autophagy, 13: 3-23.

Frischmeyer, P. A., A. van Hoof, K. O'Donnell, A. L. Guerrerio, R. Parker, and H. C. Dietz. 2002. 'An mRNA surveillance mechanism that eliminates transcripts lacking termination codons', Science, 295: 2258-61.

This article is protected by copyright. All rights reserved. 
Gatica, D., V. Lahiri, and D. J. Klionsky. 2018. 'Cargo recognition and degradation by selective autophagy', Nat Cell Biol, 20: 233-42.

Gibbings, D., S. Mostowy, F. Jay, Y. Schwab, P. Cossart, and O. Voinnet. 2012. 'Selective autophagy degrades DICER and AGO2 and regulates miRNA activity', Nat Cell Biol, 14: 1314-21.

Gomez-Sanchez, R., J. Rose, R. Guimaraes, M. Mari, D. Papinski, E. Rieter, W. J. Geerts, R. Hardenberg, C. Kraft, C. Ungermann, and F. Reggiori. 2018. 'Atg9 establishes Atg2dependent contact sites between the endoplasmic reticulum and phagophores', J Cell Biol.

Grumati, P., and I. Dikic. 2018. 'Ubiquitin signaling and autophagy', J Biol Chem, 293: 540413.

Guimaraes, R. S., E. Delorme-Axford, D. J. Klionsky, and F. Reggiori. 2015. 'Assays for the biochemical and ultrastructural measurement of selective and nonselective types of autophagy in the yeast Saccharomyces cerevisiae', Methods, 75: 141-50.

Guo, H., M. Chitiprolu, D. Gagnon, L. Meng, C. Perez-Iratxeta, D. Lagace, and D. Gibbings. 2014. 'Autophagy supports genomic stability by degrading retrotransposon RNA', Nat Commun, 5: 5276.

Hanada, T., N. N. Noda, Y. Satomi, Y. Ichimura, Y. Fujioka, T. Takao, F. Inagaki, and Y. Ohsumi. 2007. 'The Atg12-Atg5 conjugate has a novel E3-like activity for protein lipidation in autophagy', J Biol Chem, 282: 37298-302.

Harding, T. M., K. A. Morano, S. V. Scott, and D. J. Klionsky. 1995. 'Isolation and characterization of yeast mutants in the cytoplasm to vacuole protein targeting pathway', J Cell Biol, 131: 591-602.

He, C., M. C. Bassik, V. Moresi, K. Sun, Y. Wei, Z. Zou, Z. An, J. Loh, J. Fisher, Q. Sun, S. Korsmeyer, M. Packer, H. I. May, J. A. Hill, H. W. Virgin, C. Gilpin, G. Xiao, R. BasselDuby, P. E. Scherer, and B. Levine. 2012. 'Exercise-induced BCL2-regulated autophagy is required for muscle glucose homeostasis', Nature, 481: 511-5.

He, F., and A. Jacobson. 2015. 'Nonsense-Mediated mRNA Decay: Degradation of Defective Transcripts Is Only Part of the Story', Annu Rev Genet, 49: 339-66.

Heck, A. M., and J. Wilusz. 2018. 'The Interplay between the RNA Decay and Translation Machinery in Eukaryotes', Cold Spring Harb Perspect Biol, 10.

Hu, G., T. McQuiston, A. Bernard, Y. D. Park, J. Qiu, A. Vural, N. Zhang, S. R. Waterman, N. H. Blewett, T. G. Myers, R. J. Maraia, J. H. Kehrl, G. Uzel, D. J. Klionsky, and P. R. Williamson. 2015. 'A conserved mechanism of TOR-dependent RCK-mediated mRNA degradation regulates autophagy', Nat Cell Biol, 17: 930-42.

Huang, H., T. Kawamata, T. Horie, H. Tsugawa, Y. Nakayama, Y. Ohsumi, and E. Fukusaki. 2015. 'Bulk RNA degradation by nitrogen starvation-induced autophagy in yeast', Embo j, 34: 154-68.

Huch, S., and T. Nissan. 2014. 'Interrelations between translation and general mRNA degradation in yeast', Wiley Interdiscip Rev RNA, 5: 747-63.

This article is protected by copyright. All rights reserved. 
Hutchins, M. U., M. Veenhuis, and D. J. Klionsky. 1999. 'Peroxisome degradation in Saccharomyces cerevisiae is dependent on machinery of macroautophagy and the Cvt pathway', J Cell Sci, 112 ( Pt 22): 4079-87.

Ichimura, Y., T. Kirisako, T. Takao, Y. Satomi, Y. Shimonishi, N. Ishihara, N. Mizushima, I. Tanida, E. Kominami, M. Ohsumi, T. Noda, and Y. Ohsumi. 2000. 'A ubiquitin-like system mediates protein lipidation', Nature, 408: 488-92.

Jackson, W. T., T. H. Giddings, Jr., M. P. Taylor, S. Mulinyawe, M. Rabinovitch, R. R. Kopito, and K. Kirkegaard. 2005. 'Subversion of cellular autophagosomal machinery by RNA viruses', PLoS Biol, 3: e156.

Jin, M., D. He, S. K. Backues, M. A. Freeberg, X. Liu, J. K. Kim, and D. J. Klionsky. 2014. 'Transcriptional regulation by Pho23 modulates the frequency of autophagosome formation', Curr Biol, 24: 1314-22.

Kabeya, Y., N. Mizushima, T. Ueno, A. Yamamoto, T. Kirisako, T. Noda, E. Kominami, Y. Ohsumi, and T. Yoshimori. 2000. 'LC3, a mammalian homologue of yeast Apg8p, is localized in autophagosome membranes after processing', Embo j, 19: 5720-8.

Kanki, T., K. Wang, Y. Cao, M. Baba, and D. J. Klionsky. 2009. 'Atg32 is a mitochondrial protein that confers selectivity during mitophagy', Dev Cell, 17: 98-109.

Kelly, S. P., and D. M. Bedwell. 2015. 'Both the autophagy and proteasomal pathways facilitate the Ubp3p-dependent depletion of a subset of translation and RNA turnover factors during nitrogen starvation in Saccharomyces cerevisiae', Rna, 21: 898-910.

Kim, J., W. P. Huang, and D. J. Klionsky. 2001. 'Membrane recruitment of Aut7p in the autophagy and cytoplasm to vacuole targeting pathways requires Aut1p, Aut2p, and the autophagy conjugation complex', J Cell Biol, 152: 51-64.

Kirisako, T., M. Baba, N. Ishihara, K. Miyazawa, M. Ohsumi, T. Yoshimori, T. Noda, and Y. Ohsumi. 1999. 'Formation process of autophagosome is traced with Apg8/Aut7p in yeast', J Cell Biol, 147: 435-46.

Kirisako, T., Y. Ichimura, H. Okada, Y. Kabeya, N. Mizushima, T. Yoshimori, M. Ohsumi, T. Takao, T. Noda, and Y. Ohsumi. 2000. 'The reversible modification regulates the membrane-binding state of Apg8/Aut7 essential for autophagy and the cytoplasm to vacuole targeting pathway', J Cell Biol, 151: 263-76.

Klionsky, D. J., and B. A. Schulman. 2014. 'Dynamic regulation of macroautophagy by distinctive ubiquitin-like proteins', Nat Struct Mol Biol, 21: 336-45.

Kraft, C., A. Deplazes, M. Sohrmann, and M. Peter. 2008. 'Mature ribosomes are selectively degraded upon starvation by an autophagy pathway requiring the Ubp3p/Bre $5 p$ ubiquitin protease', Nat Cell Biol, 10: 602-10.

Kristensen, A. R., S. Schandorff, M. Hoyer-Hansen, M. O. Nielsen, M. Jaattela, J. Dengjel, and J. S. Andersen. 2008. 'Ordered organelle degradation during starvation-induced autophagy', Mol Cell Proteomics, 7: 2419-28.

This article is protected by copyright. All rights reserved. 
Kuma, A., N. Mizushima, N. Ishihara, and Y. Ohsumi. 2002. 'Formation of the approximately 350-kDa Apg12-Apg5.Apg16 multimeric complex, mediated by Apg16 oligomerization, is essential for autophagy in yeast', J Biol Chem, 277: 18619-25.

Kwon, Y. T., and A. Ciechanover. 2017. 'The Ubiquitin Code in the Ubiquitin-Proteasome System and Autophagy', Trends Biochem Sci, 42: 873-86.

Labno, A., R. Tomecki, and A. Dziembowski. 2016. 'Cytoplasmic RNA decay pathways Enzymes and mechanisms', Biochim Biophys Acta, 1863: 3125-47.

Liang, X. H., S. Jackson, M. Seaman, K. Brown, B. Kempkes, H. Hibshoosh, and B. Levine. 1999. 'Induction of autophagy and inhibition of tumorigenesis by beclin 1', Nature, 402: 672-6.

Liu, X., K. Mao, A. Y. H. Yu, A. Omairi-Nasser, J. Austin, 2nd, B. S. Glick, C. K. Yip, and D. J. Klionsky. 2016. 'The Atg17-Atg31-Atg29 Complex Coordinates with Atg11 to Recruit the Vam7 SNARE and Mediate Autophagosome-Vacuole Fusion', Curr Biol, 26: 15060.

Losson, R., and F. Lacroute. 1979. 'Interference of nonsense mutations with eukaryotic messenger RNA stability', Proc Natl Acad Sci U S A, 76: 5134-7.

Lubas, M., L. M. Harder, C. Kumsta, I. Tiessen, M. Hansen, J. S. Andersen, A. H. Lund, and L. B. Frankel. 2018. 'eIF5A is required for autophagy by mediating ATG3 translation', EMBO Rep, 19.

Lykke-Andersen, S., and T. H. Jensen. 2015. 'Nonsense-mediated mRNA decay: an intricate machinery that shapes transcriptomes', Nat Rev Mol Cell Biol, 16: 665-77.

Lynch-Day, M.A., and D.J. Klionsky. 2010. 'The Cvt pathway as a model for selective autophagy', FEBS Lett, 584: 1359-66.

Mao, K., L. H. Chew, Y. Inoue-Aono, H. Cheong, U. Nair, H. Popelka, C. K. Yip, and D. J. Klionsky. 2013. 'Atg29 phosphorylation regulates coordination of the Atg17-Atg31Atg29 complex with the Atg11 scaffold during autophagy initiation', Proc Natl Acad Sci U S A, 110: E2875-84.

Mercer, T. J., A. Gubas, and S. A. Tooze. 2018. 'A molecular perspective of mammalian autophagosome biogenesis', J Biol Chem, 293: 5386-95.

Mizushima, N., T. Noda, and Y. Ohsumi. 1999. 'Apg16p is required for the function of the Apg12p-Apg5p conjugate in the yeast autophagy pathway', Embo j, 18: 3888-96.

Mizushima, N., H. Sugita, T. Yoshimori, and Y. Ohsumi. 1998. 'A new protein conjugation system in human. The counterpart of the yeast Apg12p conjugation system essential for autophagy', J Biol Chem, 273: 33889-92.

Mochida, K., Y. Oikawa, Y. Kimura, H. Kirisako, H. Hirano, Y. Ohsumi, and H. Nakatogawa. 2015. 'Receptor-mediated selective autophagy degrades the endoplasmic reticulum and the nucleus', Nature, 522: 359-62.

Mordier, S., C. Deval, D. Bechet, A. Tassa, and M. Ferrara. 2000. 'Leucine limitation induces autophagy and activation of lysosome-dependent proteolysis in C2C12 myotubes

This article is protected by copyright. All rights reserved. 
through a mammalian target of rapamycin-independent signaling pathway', J Biol Chem, 275: 29900-6.

Nair, U., W. L. Yen, M. Mari, Y. Cao, Z. Xie, M. Baba, F. Reggiori, and D. J. Klionsky. 2012. 'A role for Atg8-PE deconjugation in autophagosome biogenesis', Autophagy, 8: 780-93.

Noda, T., and Y. Ohsumi. 1998. 'Tor, a phosphatidylinositol kinase homologue, controls autophagy in yeast', J Biol Chem, 273: 3963-6.

Okamoto, K., N. Kondo-Okamoto, and Y. Ohsumi. 2009. 'Mitochondria-anchored receptor Atg32 mediates degradation of mitochondria via selective autophagy', Dev Cell, 17: 87-97.

Ossareh-Nazari, B., M. Bonizec, M. Cohen, S. Dokudovskaya, F. Delalande, C. Schaeffer, A. Van Dorsselaer, and C. Dargemont. 2010. 'Cdc48 and Ufd3, new partners of the ubiquitin protease Ubp3, are required for ribophagy', EMBO Rep, 11: 548-54.

Ossareh-Nazari, B., C. A. Nino, M. H. Bengtson, J. W. Lee, C. A. Joazeiro, and C. Dargemont. 2014. 'Ubiquitylation by the Ltn $1 \mathrm{E} 3$ ligase protects $60 \mathrm{~S}$ ribosomes from starvationinduced selective autophagy', J Cell Biol, 204: 909-17.

Parker, R. 2012. 'RNA degradation in Saccharomyces cerevisae', Genetics, 191: 671-702.

Parzych, K. R., and D. J. Klionsky. 2014. 'An overview of autophagy: morphology, mechanism, and regulation', Antioxid Redox Signal, 20: 460-73.

Ponpuak, M., M. A. Mandell, T. Kimura, S. Chauhan, C. Cleyrat, and V. Deretic. 2015. 'Secretory autophagy', Curr Opin Cell Biol, 35: 106-16.

Popelka, H., and D. J. Klionsky. 2015. 'Post-translationally-modified structures in the autophagy machinery: an integrative perspective', Febs $j$, 282: 3474-88.

Presnyak, V., and J. Coller. 2013. 'The DHH1/RCKp54 family of helicases: an ancient family of proteins that promote translational silencing', Biochim Biophys Acta, 1829: 81723.

Protter, D. S., and R. Parker. 2016. 'Principles and Properties of Stress Granules', Trends Cell Biol, 26: 668-79.

Quinternet, M., M. E. Chagot, B. Rothe, D. Tiotiu, B. Charpentier, and X. Manival. 2016. 'Structural Features of the Box C/D snoRNP Pre-assembly Process Are Conserved through Species', Structure, 24: 1693-706.

Reggiori, F., and D. J. Klionsky. 2013. 'Autophagic processes in yeast: mechanism, machinery and regulation', Genetics, 194: 341-61.

Reggiori, F., T. Shintani, U. Nair, and D. J. Klionsky. 2005. 'Atg9 cycles between mitochondria and the pre-autophagosomal structure in yeasts', Autophagy, 1: 101-9.

Reggiori, F., and C. Ungermann. 2017. 'Autophagosome Maturation and Fusion', J Mol Biol, 429: 486-96.

Rojas-Rios, P., A. Chartier, S. Pierson, D. Severac, C. Dantec, I. Busseau, and M. Simonelig. 2015. 'Translational Control of Autophagy by Orb in the Drosophila Germline', Dev Cell, 35: 622-31.

This article is protected by copyright. All rights reserved. 
Rothe, B., J. M. Saliou, M. Quinternet, R. Back, D. Tiotiu, C. Jacquemin, C. Loegler, F. Schlotter, V. Pena, K. Eckert, S. Morera, A. V. Dorsselaer, C. Branlant, S. Massenet, S. SanglierCianferani, X. Manival, and B. Charpentier. 2014. 'Protein Hit1, a novel box C/D snoRNP assembly factor, controls cellular concentration of the scaffolding protein Rsa1 by direct interaction', Nucleic Acids Res, 42: 10731-47.

Rowley, P. A. 2017. 'The frenemies within: viruses, retrotransposons and plasmids that naturally infect Saccharomyces yeasts', Yeast, 34: 279-92.

Russell, R. C., Y. Tian, H. Yuan, H. W. Park, Y. Y. Chang, J. Kim, H. Kim, T. P. Neufeld, A. Dillin, and K. L. Guan. 2013. 'ULK1 induces autophagy by phosphorylating Beclin-1 and activating VPS34 lipid kinase', Nat Cell Biol, 15: 741-50.

Sakoh-Nakatogawa, M., K. Matoba, E. Asai, H. Kirisako, J. Ishii, N. N. Noda, F. Inagaki, H. Nakatogawa, and Y. Ohsumi. 2013. 'Atg12-Atg5 conjugate enhances E2 activity of Atg3 by rearranging its catalytic site', Nat Struct Mol Biol, 20: 433-9.

Sarkar, S., J. E. Davies, Z. Huang, A. Tunnacliffe, and D. C. Rubinsztein. 2007. 'Trehalose, a novel mTOR-independent autophagy enhancer, accelerates the clearance of mutant huntingtin and alpha-synuclein', J Biol Chem, 282: 5641-52.

Sawa-Makarska, J., C. Abert, J. Romanov, B. Zens, I. Ibiricu, and S. Martens. 2014. 'Cargo binding to Atg19 unmasks additional Atg8 binding sites to mediate membrane-cargo apposition during selective autophagy', Nat Cell Biol, 16: 425-33.

Scott, S. V., J. Guan, M. U. Hutchins, J. Kim, and D. J. Klionsky. 2001. 'Cvt19 is a receptor for the cytoplasm-to-vacuole targeting pathway', Mol Cell, 7: 1131-41.

Sheinberger, J., and Y. Shav-Tal. 2017. 'mRNPs meet stress granules', FEBS Lett, 591: 253442.

Shintani, T., N. Mizushima, Y. Ogawa, A. Matsuura, T. Noda, and Y. Ohsumi. 1999. 'Apg10p, a novel protein-conjugating enzyme essential for autophagy in yeast', Embo j, 18: 5234-41.

Suzuki, K., M. Morimoto, C. Kondo, and Y. Ohsumi. 2011. 'Selective autophagy regulates insertional mutagenesis by the Ty1 retrotransposon in Saccharomyces cerevisiae', Dev Cell, 21: 358-65.

Talarek, N., E. Cameroni, M. Jaquenoud, X. Luo, S. Bontron, S. Lippman, G. Devgan, M. Snyder, J. R. Broach, and C. De Virgilio. 2010. 'Initiation of the TORC1-regulated G0 program requires Igo1/2, which license specific mRNAs to evade degradation via the 5'-3' mRNA decay pathway', Mol Cell, 38: 345-55.

Tang, H. W., Y. Hu, C. L. Chen, B. Xia, J. Zirin, M. Yuan, J. M. Asara, L. Rabinow, and N. Perrimon. 2018. 'The TORC1-Regulated CPA Complex Rewires an RNA Processing Network to Drive Autophagy and Metabolic Reprogramming', Cell Metab, 27: 104054.e8.

Tanida, I., N. Mizushima, M. Kiyooka, M. Ohsumi, T. Ueno, Y. Ohsumi, and E. Kominami. 1999. 'Apg7p/Cvt2p: A novel protein-activating enzyme essential for autophagy', Mol Biol Cell, 10: 1367-79. 
Teter, S. A., K. P. Eggerton, S. V. Scott, J. Kim, A. M. Fischer, and D. J. Klionsky. 2001. 'Degradation of lipid vesicles in the yeast vacuole requires function of Cvt17, a putative lipase', J Biol Chem, 276: 2083-7.

Thorburn, A. 2018. 'Autophagy and disease', J Biol Chem, 293: 5425-30.

van Hoof, A., P. A. Frischmeyer, H. C. Dietz, and R. Parker. 2002. 'Exosome-mediated recognition and degradation of mRNAs lacking a termination codon', Science, 295: $2262-4$.

Wek, S. A., S. Zhu, and R. C. Wek. 1995. 'The histidyl-tRNA synthetase-related sequence in the eIF-2 alpha protein kinase GCN2 interacts with tRNA and is required for activation in response to starvation for different amino acids', Mol Cell Biol, 15: 4497-506.

Wen, X., and D. J. Klionsky. 2016. 'An overview of macroautophagy in yeast', J Mol Biol, 428: 1681-99.

Wengrod, J., L. Martin, D. Wang, P. Frischmeyer-Guerrerio, H. C. Dietz, and L. B. Gardner. 2013. 'Inhibition of nonsense-mediated RNA decay activates autophagy', Mol Cell Biol, 33: 2128-35.

Wong, J., J. Zhang, X. Si, G. Gao, I. Mao, B. M. McManus, and H. Luo. 2008. 'Autophagosome supports coxsackievirus B3 replication in host cells', J Virol, 82: 9143-53.

Wyant, G. A., M. Abu-Remaileh, E. M. Frenkel, N. N. Laqtom, V. Dharamdasani, C. A. Lewis, S. H. Chan, I. Heinze, A. Ori, and D. M. Sabatini. 2018. 'NUFIP1 is a ribosome receptor for starvation-induced ribophagy', Science, 360: 751-58.

Xie, Z., U. Nair, and D. J. Klionsky. 2008. 'Atg8 controls phagophore expansion during autophagosome formation', Mol Biol Cell, 19: 3290-8.

Yang, Z., J. Huang, J. Geng, U. Nair, and D. J. Klionsky. 2006. 'Atg22 recycles amino acids to link the degradative and recycling functions of autophagy', Mol Biol Cell, 17: 5094104.

Yorimitsu, T., and D. J. Klionsky. 2005. 'Atg11 links cargo to the vesicle-forming machinery in the cytoplasm to vacuole targeting pathway', Mol Biol Cell, 16: 1593-605.

Yorimitsu, T., S. Zaman, J. R. Broach, and D. J. Klionsky. 2007. 'Protein kinase A and Sch9 cooperatively regulate induction of autophagy in Saccharomyces cerevisiae', Mol Biol Cell, 18: 4180-9.

Yu, L., Y. Chen, and S. A. Tooze. 2018. 'Autophagy pathway: Cellular and molecular mechanisms', Autophagy, 14: 207-15.

Figure 1. An overview of autophagy in the yeast Saccharomyces cerevisiae. Following induction by either starvation or treatment with rapamycin, inactivation of TOR triggers

This article is protected by copyright. All rights reserved. 
autophagy. TOR/MTOR is a serine/threonine kinase and major negative regulator of autophagy (Noda and Ohsumi 1998). In a stepwise mechanism in yeast, the phagophore nucleates at the perivacuolar phagophore assembly site (PAS). The phagophore expands to envelope proximal cytosolic cargo; this process requires 2 conserved Ubl-conjugation systems: Atg12 (consisting of Atg5, Atg7, Atg10, Atg12 and Atg16) and Atg8 (including Atg3, Atg4, Atg7 and Atg8). The phagophore closes and matures to form the complete double-membrane autophagosome. Atg4 has multiple roles, including proteolytic processing of Atg8 at its $C$ terminus during early autophagy. A second Atg4-mediated cleavage event subsequently deconjugates Atg8 from the autophagosome outer membrane (Kirisako et al. 2000; Nair et al. 2012). Fusion of the autophagosome with the vacuole is mediated by proteins such as the SNARE Vam7 (Liu et al. 2016). Atg15 is required for the degradation of autophagic body membranes, allowing subsequent degradation of the sequestered cargo by vacuolar hydrolases. The current data demonstrate an efflux model for amino acids into the cytosol (Yang et al. 2006); however, products resulting from RNA catabolism are released from the cell (Huang et al. 2015), and the mechanisms used for the efflux of metabolic byproducts of other macromolecules (carbohydrates and lipids) have yet to be identified.

Figure 2. Canonical mRNA decay pathways. RNA decay pathways are essential for regulating gene expression post-transcriptionally. The 2 major pathways controlling mRNA degradation occur in either the $5^{\prime}$ to $3^{\prime}$ (left) or the $3^{\prime}$ to $5^{\prime}$ (right) direction by the multisubunit exosome. During canonical 5' to 3' RNA decay, transcripts undergo deadenylation and

This article is protected by copyright. All rights reserved. 
decapping by the Dcp2 catalytic complex followed by Xrn1-mediated hydrolysis. After 3' deadenylation, the multisubunit exosome complex degrades transcripts in the 3 ' to 5 ' direction.

Figure 3. ATG regulation by 5' to 3' mRNA decay. (A) During nutrient-rich conditions in yeast, TORC1 performs multiple roles in autophagy inhibition. Rim15 (a positive regulator of autophagy) is inhibited by major cellular nutrient sensors TORC1 and protein kinase A (PKA). TORC1 phosphorylates the catalytic decapping factor Dcp2. Dcp2 (in conjuction with Dcp1) negatively regulates autophagy by targeting ATG transcripts for decapping. At the same time, the decapping stimulatory factor Dhh1 and the exonuclease Xrn1 negatively regulate select autophagy transcripts. (B) Autophagy induction by nutrient-starvation (or rapamycin treatment for Igo1/2) inactivates TORC1, enabling Rim15 to phosphorylate Igo1/2. Igo1/2 phosphorylation promotes its asociation with Dhh1, thereby protecting transcripts from RNA decay. Whether Igo1/2 associates with Dhh1 to influence ATG transcripts during nitrogen starvation remains to be investigated. Canonical 5' to 3' RNA decay components Dcp2 and Xrn1 are downregulated during nitrogen starvation. During starvation, select ATG mRNAs (which are targeted for decay by Dhh1, Dcp2 and Xrn1 under nutrient-replete conditions) are no longer marked for degradation and are instead likely translated to promote autophagy. For clarity, the physical interaction between Dcp2 and the C-terminal region of Dhh1 (Decker, Teixeira, and Parker 2007 ) is not depicted here.

\section{Table 1. Core Atg/ATG proteins involved in autophagosome biogenesis.}

This article is protected by copyright. All rights reserved. 

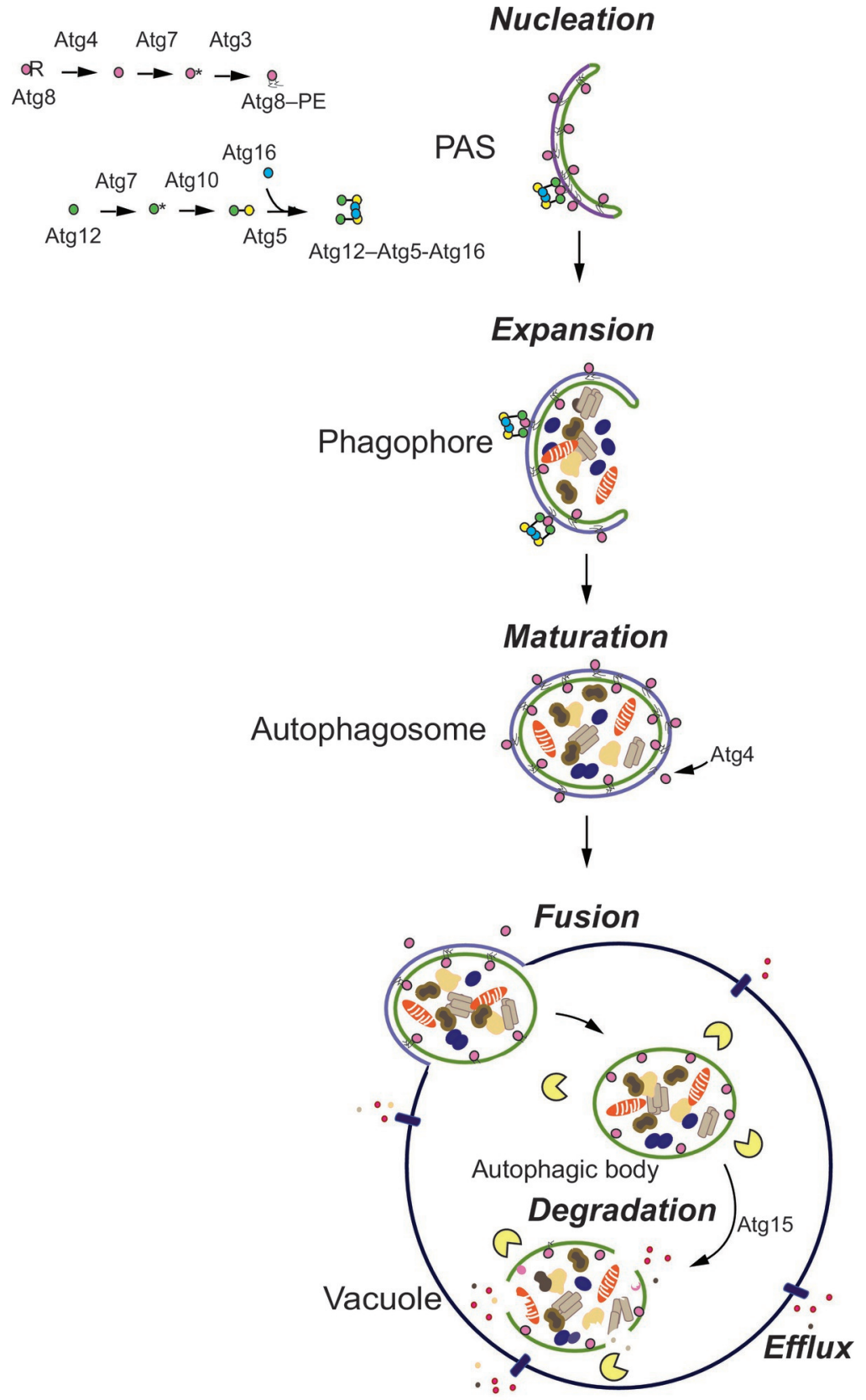

fig1 11-21-18.eps

This article is protected by copyright. All rights reserved. 

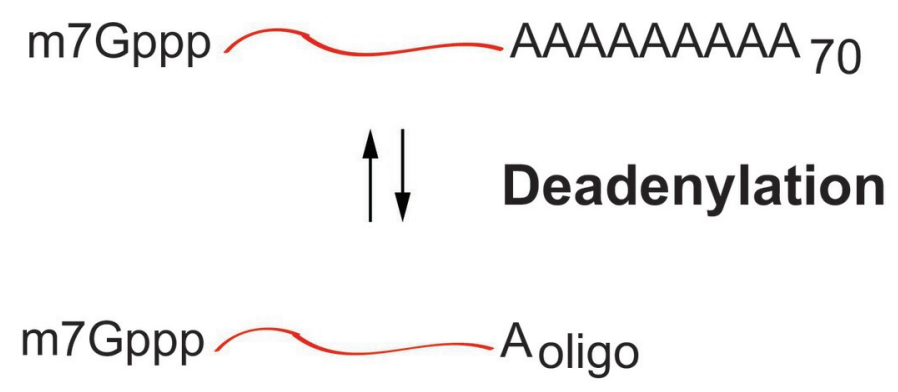

Dhh1



Dcp2
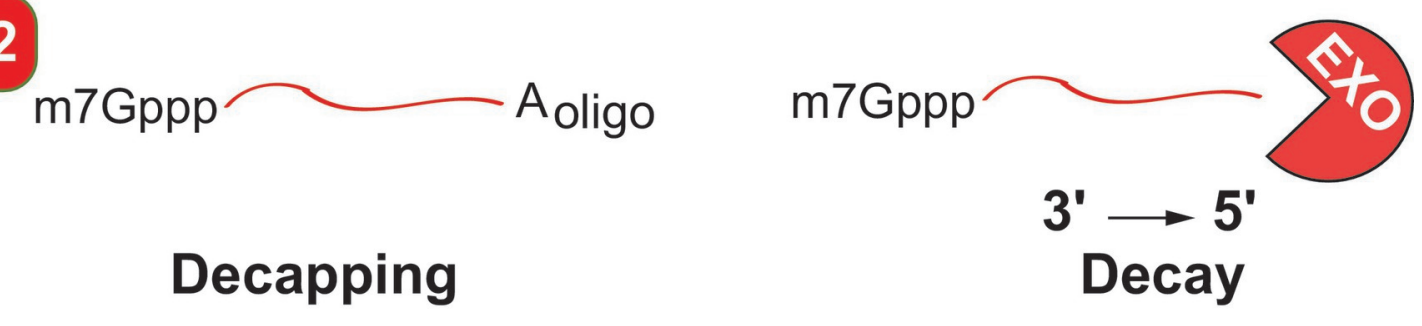

Decapping

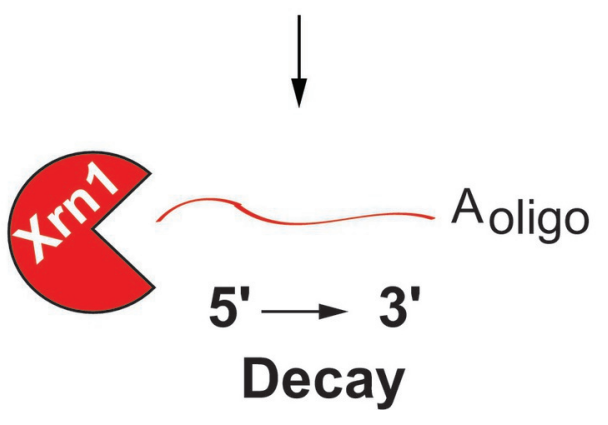

fig2-decay2 11-21-18.eps 


\section{A Nutrient-rich $\longrightarrow$ Autophagy}

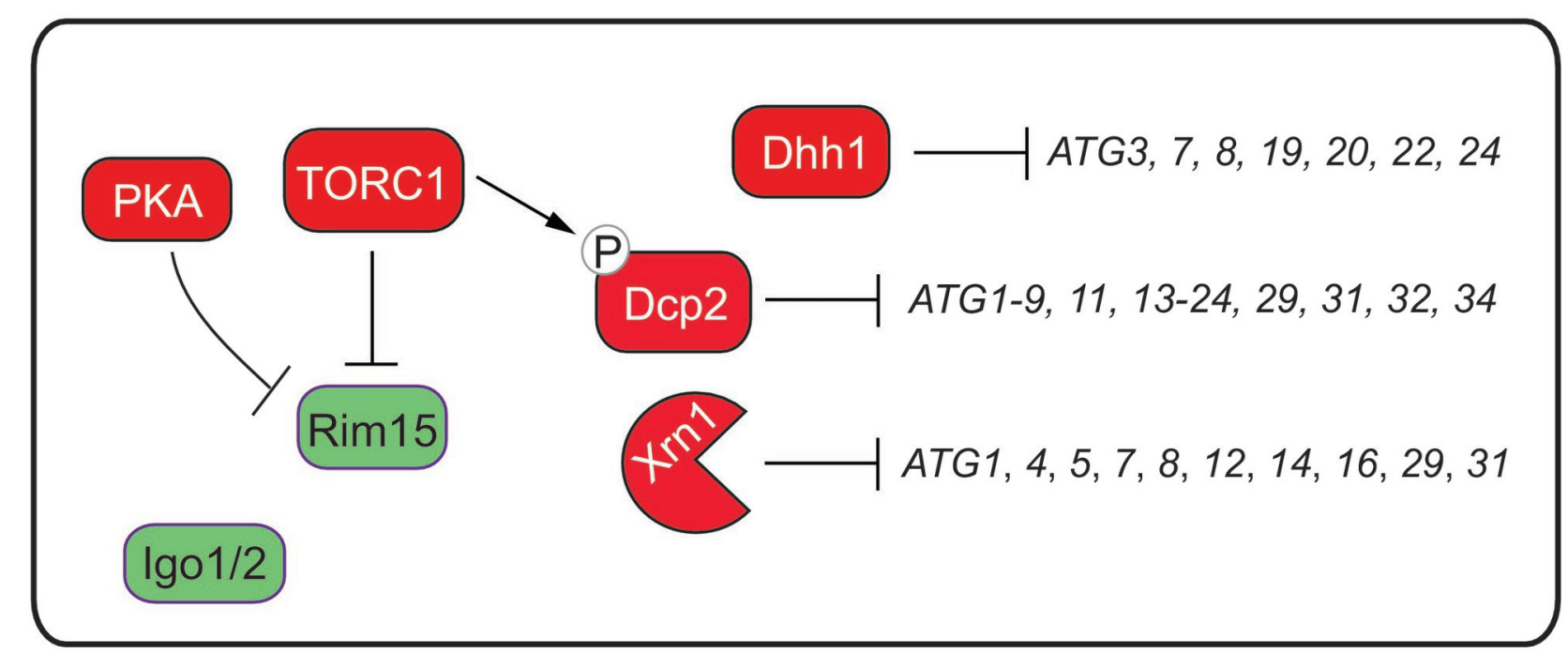

B Starvation $\longrightarrow$ Autophagy



fig3rev-11-21-18.eps 


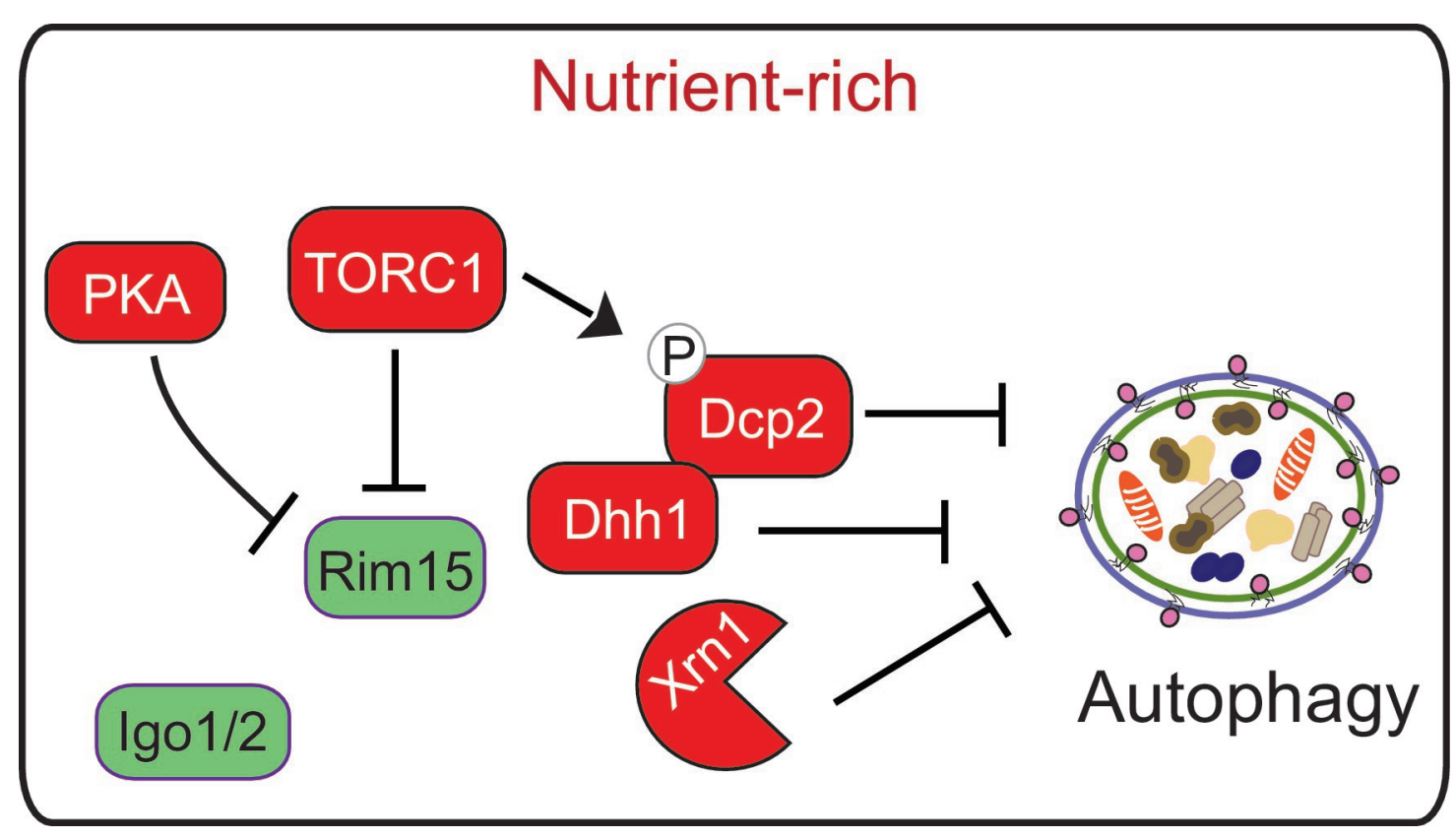

ga11-21-18.eps

This article is protected by copyright. All rights reserved. 


\begin{tabular}{|c|c|c|c|}
\hline & Yeast & Mammals & \\
\hline \multirow[t]{9}{*}{ Atg1 complex } & Atg1 & ULK1 & Induction \\
\hline & Atg11 & & \\
\hline & Atg13 & ATG13 & \\
\hline & Atg17 & RB1CC1/FIP200 & \\
\hline & Atg20 & & \\
\hline & Atg24 & & \\
\hline & Atg29 & & \\
\hline & Atg31 & & \\
\hline & & ATG101 & \\
\hline \multirow[t]{5}{*}{ Ptdlns3K complex } & Vps34 & PIK3C3/VPS34 & Nucleation \\
\hline & Vps15 & PIK3R4/p150/VPS15 & \\
\hline & Vps30/Atg6 & BECN1 & \\
\hline & Atg14 & ATG14/Barkor & \\
\hline & Atg38 & NRBF2 & \\
\hline \multirow[t]{4}{*}{ Atg9 and cycling system } & Atg9 & ATG9A/B & Membrane donation \\
\hline & $\operatorname{Atg} 2$ & ATG2 & \\
\hline & Atg18 & WIPI1/2 & \\
\hline & Atg41 & & \\
\hline \multirow[t]{4}{*}{ Atg8 Ubl conjugation system } & Atg8 & $\begin{array}{c}\text { LC3A/B/B2/C } \\
\text { GABARAP/L1/L2 }\end{array}$ & Expansion \\
\hline & Atg3 & ATG3 & \\
\hline & Atg 4 & ATG4A/B/C/D & \\
\hline & Atg7 & ATG7 & \\
\hline \multirow[t]{5}{*}{ Atg12 Ubl conjugation system } & Atg12 & ATG12 & Expansion \\
\hline & Atg5 & ATG5 & \\
\hline & $\operatorname{Atg} 7$ & ATG7 & \\
\hline & Atg10 & ATG10 & \\
\hline & Atg16 & ATG16L1 & \\
\hline
\end{tabular}

table1-atg-071618.eps

This article is protected by copyright. All rights reserved. 